\title{
HARMONIC MEASURE AND DOMAINS BOUNDED BY QUASICONFORMAL CIRCLES ${ }^{1}$
}

\author{
DONALD K. BLEVINS
}

\begin{abstract}
We index the class of quasiconformal circles by $k$ between zero and one such that $k=0$ corresponds to arbitrary Jordan curves and $k=1$ to circles. We establish an estimate depending on $k$ for harmonic measure in a domain bounded by a quasiconformal circle. Applications of this estimate are made to boundary correspondence under conformal maps, Hardy class of certain functions and a Phragmén-Lindelöf theorem.
\end{abstract}

1. A Jordan curve $\Gamma$ in $\bar{C}$ is called a quasiconformal circle if there is a quasiconformal map $f$ of a neighborhood $V$ of the unit circle $S$ into $C$ such that $\Gamma=f(S)$. Ahlfors [1] proved the following necessary and sufficient metric condition that $\Gamma$ be a quasiconformal circle. We designate the chordal distance between points $z_{1}, z_{2} \in \bar{C}$ by $q\left(z_{1}, z_{2}\right)$ and define the chordal cross ratio of the quadruple $z_{1}, z_{2}, z_{3}, z_{4}$ in $\bar{C}$ by

$$
X\left(z_{1}, z_{2}, z_{3}, z_{4}\right)=\frac{q\left(z_{1}, z_{2}\right) q\left(z_{3}, z_{4}\right)}{q\left(z_{1}, z_{3}\right) q\left(z_{2}, z_{4}\right)} .
$$

Ahlfor's condition then is that $\Gamma$ is a quasiconformal circle if and only if $\sup \left(X\left(z_{1}, z_{2}, z_{3}, z_{4}\right)+X\left(z_{2}, z_{3}, z_{4}, z_{1}\right)\right)$ is finite, where the supremum is taken over all ordered quadruples $z_{1}, z_{2}, z_{3}, z_{4}$ on $\Gamma$.

Based on this condition, we say a Jordan curve $\Gamma$ is a $k$-circle, where $0<k \leqq 1$, if

$$
X\left(z_{1}, z_{2}, z_{3}, z_{4}\right)+X\left(z_{2}, z_{3}, z_{4}, z_{1}\right) \leqq 1 / k
$$

for all ordered quadruples $z_{1}, z_{2}, z_{3}, z_{4}$ on $\Gamma$. By the obvious extension of (1.1) we say an arbitrary Jordan curve is a 0 -circle.

Note that the left-hand side of (1.1) is always greater than or equal to one, and a 1-circle is a Euclidean circle or straight line. Thus the class of $k$-circles, as $k$ runs from zero to one, in some sense interpolates between

Received by the editors February 19, 1973.

AMS (MOS) subject classifications (1970). Primary 31A05, 30A44; Secondary 30A78, 31A20.

Key words and phrases. Quasiconformal circles, harmonic measure.

1 This work is partially excerpted from the author's Ph.D. thesis written at the University of Michigan under Professor F. W. Gehring.

(c) American Mathematical Society 1973 
arbitrary Jordan curves and the simplest Jordan curves, circles. The results in this paper illustrate some aspects of this interpolation property.

In the following we will discuss some properties of domains bounded by $k$-circles. We call such a domain a $k$-domain. In $\$ 2$, we derive a basic estimate for harmonic measure on a $k$-domain, and in $\$ 3$ we apply this result to obtain results on boundary correspondence under conformal maps, a Phragmén-Lindelöf type theorem, and results on the Hardy class of certain functions.

Before continuing we establish some notation and terminology. $B\left(z_{0}, r\right)$ is the open disk $\left\{z:\left|z-z_{0}\right|<r\right\}, B(r)=B(0, r)$ and $B=B(1) . S\left(z_{0}, r\right)$ is the boundary of $B\left(z_{0}, r\right), S(r)=S(0, r)$ and $S=S(1)$. The complement of a set $D$ is denoted by $\sim D$. A domain $R$ with two complementary components is called a ring domain or simply a ring. Such a domain is conformally equivalent to the punctured plane, a punctured disk, or an annulus $\{z: a<|z|<b\}$. We define the modulus of $R, \operatorname{Mod}(R)$, in the first two cases to be infinite and in the last case to be $\log (b / a)$. We will use the technique of circular symmetrization and the fact that the modulus of a ring is not decreased by symmetrization [3].

2. In this section we develop an estimate for harmonic measure on a $k$-domain. We start with the following lemma.

Lemma 2.1. Let $\Gamma$ be a $k$-circle with $0, \infty \in \Gamma$. Let $D$ be a domain contained in $\sim \operatorname{cl}(B(d))$ bounded by one arc $C$ of $S(d)$ and a closed subarc $\Gamma_{1}$ of $\Gamma$ with $\infty \in \Gamma_{1}$. Let $z_{0} \in D$ and designate the harmonic measure of $C$ at $z_{0}$ with respect to $D$ by $\omega\left(z_{0}, C ; D\right)$. Then

$$
\omega\left(z_{0}, C ; D\right) \leqq(4 / \pi) \arctan \left(\left(d /\left|z_{0}\right|\right)^{\pi / 2(\pi-\arcsin k)}\right) .
$$

Proof. Let $f$ be the conformal map of $D$ onto $B$ normalized such that $f\left(z_{0}\right)=0$ and $C$ corresponds to $\left\{e^{i \theta}:|\theta|<\pi \omega\right\}$ where $\omega=\omega\left(z_{0}, C ; D\right)$. Let $I^{\prime}$ be the interval $[0,1]$ and $I$ be the pre-image under $f$ of $I^{\prime}$. Now $f$ can be extended by reflection in $C$ to a map which we will continue to denote by $f$ which maps $(D \cup C \cup \hat{D}) \sim(I \cup I)=R$ conformally onto $C \sim\left([0,+\infty] \cup\left\{e^{i \theta}: \pi \omega \leqq \theta \leqq 2 \pi-\pi \omega\right\}\right)=A$, where ${ }^{\wedge}$ denotes reflection in $C$. Thus $\operatorname{Mod}(R)=\operatorname{Mod}(A)$.

We now form the ring $R_{1}$ which is the circular symmetrization $R$ with respect to the ray $[0,+\infty]$. The bounded component of the complement of $R$ is $I \cup\left\{\right.$ which is a continuum joining $z_{0}$ and $d^{2} / \bar{z}_{0}$. Thus the bounded component of $R_{1}$ contains the interval $\left[d^{2} /\left|z_{0}\right|,\left|z_{0}\right|\right]$.

The unbounded component of the complement of $R$, call it $G$, is bounded by $\Gamma_{1}$ and its reflection. For $r \geqq d, S(r) \cap G$ contains a subarc with endpoints $P_{1}, P_{2}$ on $\Gamma_{1}$, such that $\infty$ lies between $P_{1}$ and $P_{2}$ on $\Gamma_{1}$. Since $\Gamma_{1}$ is a subarc of the $k$-circle $\Gamma$, we may apply inequality (1.1) with $z_{1}=P_{1}$, 
$z_{2}=\infty, z_{3}=P_{2}, z_{4}=0$ to obtain $\left(\left|P_{2}\right|+\left|P_{1}\right| /\left|P_{2}-P_{1}\right|\right) \leqq 1 / k$ or

$$
\left(\left|P_{1}-P_{2}\right| / 2 r\right) \geqq k \text {. }
$$

Hence the angular separation of $P_{1}$ and $P_{2}$ is at least $2 \arcsin k$. Similarly, for $r<d, S(r) \cap G$ contains a subarc with endpoints $P_{1}, P_{2}$ on $\hat{\Gamma}_{1}$, such that 0 lies between $P_{1}, P_{2}$ on $\hat{\Gamma}_{1} . P_{1}$ and $P_{2}$ are obtained by reflection from points $\hat{P}_{1}, \hat{P}_{2}$ on $\Gamma_{1}$ and since $X\left(z_{1}, z_{2}, z_{3}, z_{4}\right)$ is invariant under reflection we again obtain inequality (2.2) and again conclude the angular separation of $P_{1}$ and $P_{2}$ is at least $2 \arcsin k$. Thus we have shown that the symmetrization $R_{1}$ of $R$ is contained in the ring $R_{2}$ with complementary components the interval $\left[d^{2} / z_{0}, z_{0}\right]$ and $\{z: \pi-\arcsin k \leqq \arg (z) \leqq \pi+\arcsin k\}$. Thus

$$
\operatorname{Mod}(A)=\operatorname{Mod}(R) \leqq \operatorname{Mod}\left(R_{1}\right) \leqq \operatorname{Mod}\left(R_{2}\right) .
$$

The first inequality is by symmetrization and the second by monotonicity of the modulus.

A branch of the function $g(z)=\left(z /\left|z_{0}\right|\right)^{\alpha}$ with $\alpha=\pi /(\pi-\arcsin k)$ maps $\boldsymbol{R}_{\mathbf{2}}$ conformally onto the ring bounded by the negative real axis and the interval $\left[\left(d /\left|z_{0}\right|\right)^{2 \alpha}, 1\right]$. We now map the ring $A$ onto a ring bounded by the negative real axis and a slit $[a, 1]$ by the following sequence of maps. A branch of $h_{1}(z)=(-z)^{1 / 2}$ maps $A$ conformally onto the ring $A_{1}$ bounded by the imaginary axis and a circular arc. Next the map $h_{2}(z)=(1-i z) /(z-i)$ maps $A_{1}$ onto the ring $A_{2}$ bounded by the imaginary axis and the interval

$$
\left[\sin \frac{1}{2} \pi \omega /\left(1+\cos \frac{1}{2} \pi \omega\right), \sin \frac{1}{2} \pi \omega /\left(1-\cos \frac{1}{2} \pi \omega\right)\right] .
$$

Finally the function $h_{3}(z)=\left(\left(1-\cos \frac{1}{2} \pi \omega\right) z / \sin \frac{1}{2} \pi \omega\right)^{2}$ maps $A_{2}$ onto the ring bounded by the negative real axis and the interval

$$
\left[\left(\left(1-\cos \frac{1}{2} \pi \omega\right) /\left(1+\cos \frac{1}{2} \pi \omega\right)\right)^{2}, 1\right] .
$$

Now (2.3) implies $\operatorname{Mod}\left(g\left(R_{2}\right)\right) \geqq \operatorname{Mod}\left(h_{3} \circ h_{2} \circ h_{1}(A)\right)$ and thus by the monotonicity of the modulus

$$
\left(d /\left|z_{0}\right|\right)^{2} \geqq\left(\left(1-\cos \frac{1}{2} \pi \omega\right) /\left(1+\cos \frac{1}{2} \pi \omega\right)\right)^{2}
$$

or solving for $\omega, \omega\left(z_{0}, C ; D\right) \leqq(4 / \pi) \arctan \left(\left(d /\left|z_{0}\right|\right)^{\pi / 2(\pi-\arcsin k)}\right)$.

We shall now use Lemma 2.1 to derive the following theorem on subharmonic functions, which in turn yields an estimate for harmonic measure.

THEOREM 2.2. Let $D$ be a k-domain with $0 \in \partial D$. Let $G$ be a domain with $G \subset D \cup B(d)$. Let $u$ be a subharmonic function defined in $G$ with $u(z) \leqq 1$ for all $z \in G$ and $\lim \sup _{z \rightarrow \zeta} u(z) \leqq 0$ for all $\zeta \in \partial G \cap \sim \operatorname{cl}(B(d))$. Then

$$
u\left(z_{0}\right) \leqq(4 / \pi) \arctan \left(\left(d /\left|z_{0}\right|\right)^{\pi / 2(\pi-\arcsin k)}\right) \quad \text { for all } z_{0} \in G
$$


Proof. We may assume $z_{0}=-l<-d<0$. Since $\infty \notin D$ there is a smallest positive $a>l$ such that $-a \in \partial D$. We may assume $a=\infty$. If not, the Möbius transformation $T(z)=z(a-l) /(a+z)$ maps 0 to $0,-l$ to $-l, a$ to $\infty$ and $T(\operatorname{cl}(B(d))) \subset \operatorname{cl}(B(d))$, and we may consider the function $u \circ T^{-1}$ on the domain $T(G)$.

Given $\varepsilon>0$ with $d+\varepsilon<l$, there exists a unique closed subarc of $\partial D$, $\Gamma_{\varepsilon}$, with $\infty \in \Gamma_{\varepsilon}$ and $\Gamma_{\varepsilon} \subset \sim \operatorname{cl}(B(d+\varepsilon))$ except for its endpoints which lie on $S(d+\varepsilon)$. Thus $\Gamma_{\varepsilon}$ is a cross cut of the domain $\sim \operatorname{cl}(B(d+\varepsilon))$ dividing it into two disjoint domains. One of these domains contains $z_{0}$; call this domain $D_{\varepsilon}$. The boundary of $D_{\varepsilon}$ consists of $\Gamma_{\varepsilon}$ and an open arc $C_{\varepsilon} \subset S(d+\varepsilon)$.

Let $\omega(z)=\omega\left(z, C_{\varepsilon} ; D_{\varepsilon}\right)$. We claim $u(z) \leqq \omega(z)$ for all $z \in G \cap D_{\varepsilon}$. Note $\partial\left(G \cap D_{\varepsilon}\right) \subset\left(\partial G \cap \bar{D}_{\varepsilon}\right) \cup C_{\varepsilon}$. For $\zeta \in C_{\varepsilon}, \omega(\zeta)=1$ while by hypothesis $u(\zeta) \leqq 1$ so $u(\zeta) \leqq \omega(\zeta)$.

For $\zeta \in\left(\partial G \cap \bar{D}_{\varepsilon}\right)$, we have by hypothesis $u(\zeta)=0$ and since the harmonic measure is always nonnegative, $u(\zeta) \leqq \omega(\zeta)$. Thus $u(\zeta) \leqq \omega(\zeta)$ for all $\zeta \in \partial\left(G \cap D_{\varepsilon}\right)$ and by the maximum principle for subharmonic functions, $u(z) \leqq \omega(z)$ for all $z \in G \cap D_{\varepsilon}$ as claimed. In particular $u\left(z_{0}\right) \leqq \omega\left(z_{0}\right)$, and

$$
\omega\left(z_{0}\right) \leqq(4 / \pi) \arctan \left(\left((d+\varepsilon) /\left|z_{0}\right|\right)^{\pi / 2(\pi-\arcsin k)}\right)
$$

by (2.1). Thus (2.4) follows as $\varepsilon$ goes to zero.

Theorem 2.2 yields the following estimate on harmonic measure in a $k$-domain. For $k=0$ this reduces to a well-known estimate due to Beurling [2], while for $k=1$ it is a rather obvious estimate for harmonic measure in a half plane.

Corollary 2.3. If $D$ is a $k$-domain and $E$ is a Borel subset of $\partial D$ with the Euclidean diameter of $E$ less than or equal to $d$ and if $z_{0} \in D$ with the Euclidean distance from $z_{0}$ to $E$ greater than or equal to $l$, then

$$
\omega\left(z_{0}, E ; D\right) \leqq(4 / \pi) \arctan \left((d / l)^{\pi / 2(\pi-\arcsin k)}\right) .
$$

Proof. We may assume $0 \in E$. We apply Theorem 2.2 with $G=D$ and $u(z)=\omega(z, E ; D)$ to obtain $(2.5)$.

3. If $f$ is a conformal map of $B$ onto a Jordan domain $D, \mathrm{~K}$. Matsumoto [4] has shown that sets on $\partial D$ with Hausdorff $\frac{1}{2}$-dimensional measure zero correspond to sets of linear measure zero on $S$. The following theorem generalizes this result.

THEOREM 3.1. Let $f$ be a homeomorphism of $\bar{B}$ onto $\bar{D}$ such that $f$ is conformal in $D$. If $D$ is a $k$-domain and $E \subset \partial D$ has Hausdorff $\pi / 2(\pi-\arcsin k)$-dimensional measure zero, then $f^{-1}(E)$ has linear measure zero. 
Proof. It suffices to show $\omega\left(0, f^{-1}(E) ; B\right)=0$, and hence by invariance of harmonic measure under conformal maps, it suffices to show $\omega(f(0), E ; D)=0$.

For convenience of notation, let $\alpha=\pi / 2(\pi-\arcsin k), l$ be the distance from $f(0)$ to $E$. Given $r, \varepsilon>0$, since $\mu_{\alpha}(E)=0$ there is a countable collection of disks $\delta_{i}$ with radii $r_{i}$ such that $r_{i}<r, E \subset \bigcup \delta_{i}$, and $\sum r_{i}^{\alpha}<\varepsilon$. Now let $E_{i}=\partial D \cap \delta_{i}$, so for each $i$, the diameter of $E_{i}$ is less than or equal to $2 r_{i}$. By Corollary 2.3,

$$
\omega\left(f(0), E_{i} ; D\right) \leqq \frac{4}{\pi} \arctan \left(\left(\frac{2 r_{i}}{l}\right)^{\alpha}\right) \leqq \frac{4}{\pi}\left(\frac{2}{l}\right)^{\alpha} r_{i}^{\alpha} .
$$

Thus

$$
\begin{aligned}
\omega\left(f(0), \partial D \cap\left(\bigcup \delta_{i}\right) ; D\right) & \leqq \sum \omega\left(f(0), E_{i} ; D\right) \\
& \leqq \frac{4}{\pi}\left(\frac{2}{l}\right)^{\alpha} \sum r_{i}^{\alpha}<\frac{4}{\pi}\left(\frac{2}{l}\right)^{\alpha} \varepsilon
\end{aligned}
$$

Since $E \subset \partial D \cap\left(\bigcup \delta_{i}\right)$, and since $\varepsilon$ is arbitrary, $E$ has measure zero, as claimed.

As a further application of the results of $\$ 2$, we consider the Hardy class of an analytic function whose range is contained in a $k$-domain. We recall the following definition of $H_{p}(D)$. If $D$ is a domain in $C$ and $f$ is a function analytic in $D$ then $f \in H_{p}(D)$ if $|f(z)|^{p}$ has a harmonic majorant in $D$.

Theorem 3.2. Let $D$ be a domain in $C$ and $D^{\prime} a k$-domain. If $f$ is analytic with $f(D) \subset D^{\prime}$ then $f \in H_{p}(D)$ for all $p$ with $0<p<\pi / 2(\pi-\arcsin k)$.

Proof. Let $\Gamma=\partial D^{\prime}$. We may assume $0, \infty \in \Gamma$. It suffices to show the function $|w|^{p}$ has a harmonic majorant $u(w)$ in $D^{\prime}$, for then $u(f(z))$ is a harmonic majorant for $|f(z)|^{p}$.

For each $r>0$ let $\omega_{r}(z)=\omega\left(z, \Gamma \cap \sim \operatorname{cl}(B(r)) ; D^{\prime}\right)$. Let $D^{\prime \prime}$ be the image of $D^{\prime}$ under $T(z)=1 / z, \Gamma^{\prime \prime}$ the corresponding image of $\Gamma \cap \sim \operatorname{cl}(B(r))$. Note $\Gamma^{\prime \prime} \subset B(1 / r)$ and $D^{\prime \prime}$ is a $k$-domain. By invariance of harmonic measure $\omega_{r}(z)=\omega\left(1 / z, \Gamma^{\prime \prime} ; D^{\prime \prime}\right)$ and thus by Theorem 2.2, setting $p_{0}=\pi / 2(\pi-\arcsin k)$,

$$
\omega_{r}(z) \leqq \frac{4}{\pi} \arctan \left(\left(\frac{|z|}{r}\right)^{p_{0}}\right) \leqq \frac{C}{r^{p_{0}}}
$$

where $C$ is a constant depending on $z$ but not on $r$. Hence for $p<p_{0}$ the integral $u(z)=p \int_{0}^{\infty} \omega_{r}(z) r^{p-1} d r$ converges and represents a harmonic function in $D^{\prime}$. 
As $z$ tends to a boundary point $\zeta$, we have $\lim _{z \rightarrow \zeta} \omega_{r}(z)=\omega_{r}(\zeta)$ where $\omega_{r}(\zeta)=0$ if $|\zeta|>r$, and $\omega_{r}(\zeta)=1$ if $|\zeta|<r$. Thus by dominated convergence

$$
\lim _{z \rightarrow \zeta} u(z)=p \int_{0}^{\infty} \omega_{r}(\zeta) r^{p-1} d r=p \int_{0}^{|\zeta|} r^{p-1} d r=|\zeta|^{p} .
$$

Thus $u(z)$ is a harmonic majorant for the subharmonic function $|z|^{p}$.

As a final application of Theorem 2.2 we prove a Phragmén-Lindelöf type theorem for $k$-domains.

THeOREM 3.3. Let $D$ be a $k$-domain with $\zeta_{0} \in \partial D, \zeta_{0} \neq \infty$. Let $u$ be a subharmonic function on $D$ with $\lim \sup _{z \rightarrow \zeta} u(z) \leqq 0$ for all $\zeta \in \partial D \sim\left\{\zeta_{0}\right\}$. Set $M\left(r, \zeta_{0}, u\right)=\sup \left\{u(z): z \in D \cap S\left(\zeta_{0}, r\right)\right\}$. If

$$
\liminf _{r \rightarrow 0} M\left(r, \zeta_{0}, u\right) r^{\pi / 2(\pi-\arcsin k)} \leqq 0
$$

then $u(z) \leqq 0$ for all $z \in D$.

Proof. We may assume $\zeta_{0}=0$. Choose $z_{0} \in D$ and for each $r<\left|z_{0}\right| / 2$, let $\gamma_{r}$ be an arc of $S(r)$ forming a cross cut of $D$ separating 0 from $z_{0}$ in $D$. Let $D_{r}$ be the domain thus formed containing $z_{0}$. Then by the two constants theorem for subharmonic functions we have

$$
u\left(z_{0}\right) \leqq M\left(r, \zeta_{0}, u\right) \omega\left(z_{0}, \gamma_{r} ; D_{r}\right) .
$$

By Theorem 2.2 we have

$$
\omega\left(z_{0}, \gamma_{r} ; D_{r}\right) \leqq(4 / \pi) \arctan \left(\left(r /\left|z_{0}\right|\right)^{\pi / 2(\pi-\arcsin k)}\right) \leqq C r^{\pi / 2(\pi-\arcsin k)}
$$

where $C$ depends on $z$ but not on $r$. Thus from (3.2) for each $r<\left|z_{0}\right| / 2$, $u\left(z_{0}\right) \leqq C M\left(r, \zeta_{0}, u\right) r^{\pi / 2(\pi-\arcsin k)}$. Thus by (3.1) $u\left(z_{0}\right) \leqq 0$ and since $z_{0}$ is arbitrary the result follows.

\section{REFERENCES}

1. L. V. Ahlfors, Quasiconformal reflections, Acta Math. 109 (1963), 291-301. MR 27 \#4921.

2. A. Beurling, Etudes sur un probleme de majorization, Thesis, Uppsala University, Uppsala, 1933.

3. J. A. Jenkins, Some uniqueness results in the theory of symmetrization, Ann. of Math. (2) 61 (1955), 106-115. MR 16, 460.

4. K. Matsumoto, On some boundary problems in the theory of conformal mappings of Jordan domains, Nagoya Math J. 24 (1964), 129-141. MR 34 \#324.

Department of Mathematics, University of Michigan, Ann Arbor, Michigan 48104

Current address: Department of Mathematics, University of Florida, Gainesville, Florida 32611 